Apidologie, 1986, 17 (2), 107-124

\title{
LES INSECTES POLLINISATEURS DE QUELQUES OMBELLIFĖRES D'INTÉRÊT AGRICOLE ET CONDIMENTAIRE (ANGELICA ARCHANGELICA L., CARUM CARVI L., PETROSELINUM CRISPUM A.W. HILL., APIUM GRAVEOLENS L., PIMPINELLA ANISUM L., DAUCUS CAROTA L., FOENICULUM VULGARE MILLER V. AZORICUM THELL.) *
}

\author{
Gian Carlo RICCIARDELLI D'ALBORE \\ Istituto di Entomologia Agraria, Borgo XX Giugno \\ Università di Perugia (Italie)
}

RESUMÉ

\begin{abstract}
Dans la pollinisation de quelques Ombellifères d'intérêt agricole et condimentaire les pollinisateurs sauvages joucnt un rôle essentiel. Les Diptères (Syrphidae, Muscidae, Calliphoridae) représentent $29,71 \%$ sur l'angélique (Angelica archangelica L.), $27,35 \%$ sur le carvi (Carum carvi L.), 40,73\% sur le persil (Petroselinum crispum A.W. Hill.), 44,74\% sur le céleri (Apium graveolens L.), 23,61\% sur l'anis (Pimpinella anisum L.), 9,69\% sur la carotte (Daucus carota L.) et 25,64\% sur le fenouil (Foeniculum vulgare Miller v. azoricum Thell.); Andrena minutula K. respectivement $32,70 \%, 61,40 \%, 40,89 \%, 32,07 \%, 58,74 \%, 61,55 \%$ et $33,00 \%$. Avec d'autres insectes pollinisateurs ils représentent dans l'ensemble et respectivement pour chaque Ombellifère $82,76 \%, 98,76 \%, 99,84 \%, 99,47 \%, 98,12 \%, 99,80 \%$ et $97,92 \%$ des pollinisateurs. Par contre Apis mellifera ligustica Spin. ne joue un rôle efficace que dans la pollinisation d'Angelica (représentativité $=17,24 \%$ ), les autres Ombellifères ne se montrant pas attractives.
\end{abstract}

\section{INTRODUCTION}

Dans la plupart des cas les Ombellifères sont protandriques et il leur faut donc des agents transporteurs du pollen sur les stigmates réceptifs. Il y a aussi des cas de protogynie ou même de stérilité mâle (ex. quelques variétés de Daucus carota) (Pesson et Louveaux, 1984). Le vent aussi peut apporter une contribution, du reste relative, au transport du pollen, mais en règle générale les insectes 
répondent mieux à ce but, en particulier ceux qui recueillent seulement du pollen ; toutefois ceux qui recueillent seulement du nectar peuvent jouer aussi un rôle considérable en ce qui concerne la fécondation croisée. Parmi les espèces considérées il y a longtemps que les variétés de Daucus carota, peu ou pas visitées par les abeilles, sont l'objet d'observations (FrEE, 1970) ; on a peu de renseignements à propos de Foeniculum vulgare (NARAJANAN et al., 1960). Angelica archangelica a des propriétés carminatives, aromatisantes et antispasmodiques, Carum carvi des propriétés digestives, diurétiques et sudorifiques et Pimpinella anisum des propriétés semblables aux précédentes (Pomini, 1973), Petroselinum, Apium, Daucus et Foeniculum, elles aussi dotées de vertus thérapeutiques, doivent être considérées surtout pour leur intérêt agricole et horticole.

Le but principal de ce travail est de connaître les principaux insectes pollinisateurs des Ombellifères ; un autre but est de mettre en évidence la flore compétitive qui concurrence les cultures à polliniser.

\title{
MATERIEL ET 'METHODES
}

\begin{abstract}
On a opéré dans un milieu caractérisé par des pratiques agronomiques traditionnclles où l'on n'emploie pas de produits phytosanitaires; les espèces sont cultivées depuis plusieurs années sur les mêmes parcelles d'une dimension comprise entre 10 et $18 \mathrm{~m}^{2}$ chacune, en créant pratiquement un habitat stable. A la suite de recherches préliminaires (RICCIARdelli d'Albore, 1982-1983) on a compté tous les deux jours en 1984 les insectes présents sur $1 \mathrm{~m}^{2}$ de surface; chaque jour d'observation on a capturé à trois reprises, sur 5 fois $1 \mathrm{~m}^{2}$ de chaque culture, tous les insectes qui ont été ensuite classés; les données ont été rapportées à $10 \mathrm{~m}^{2}$ de surface.

Pour étudier le déroulement de la floraison on a calculé chaque jour d'observation, et pour chaque espèce botanique, les fleurs en anthèse des ombelles primaires et successivement des ombelles secondaires de 20 plantes choisies au hasard sur les différentes parcelles. Sur la base de ce calcul, rapporté au total des fleurs des plantes examinées, on a pu tirer un diagramme suffisamment fiable par rapport au déroulement de chaque floraison.
\end{abstract}

\section{RÉSULTATS}

On a obtenu les résultats suivants :

1. Angelica archangelica L. (surface totale $15 \mathrm{~m}^{2}$ ) (Fig. 1).

Les Syrphidae montrent une fréquence de visites de 9 jours sur 20, une représentativité médiocre $(7,53 \%)$ et une densité moyenne de 13,05 butineuses.

Les Muscidae et Calliphoridae montrent une fréquence de visites remarquable $(100 \%)$, des pointes d'intensité considérables du 10 au 16 mai et une excep- 


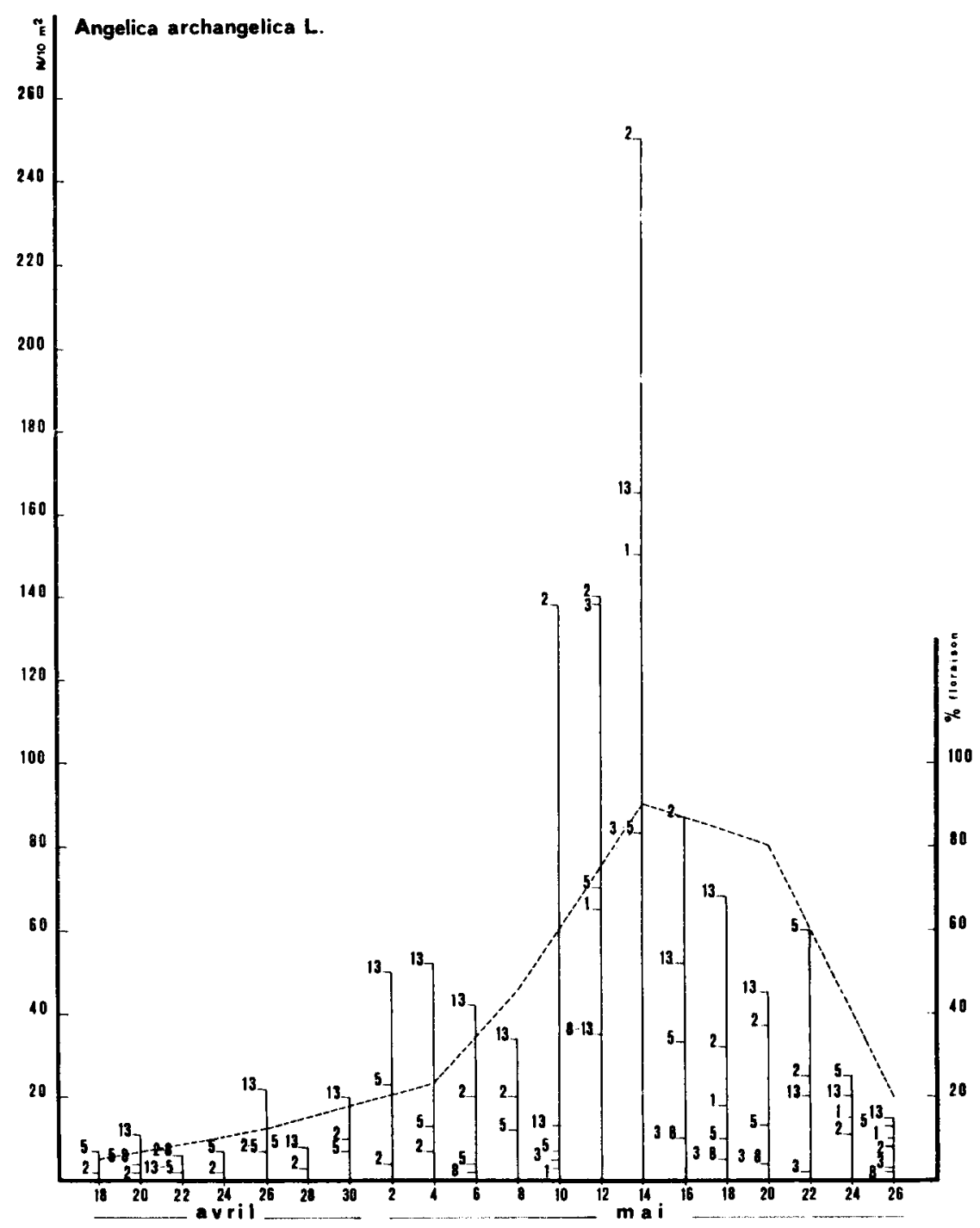

FIG. 1. - Densité de Syrphidae (1), Muscidae et Calliphoridae (2), Vespa germanica L. (3), Andrena minutula $K$. (5), Halictus maculatus $S$ mith (8) et Apis mellifica ligustica $S p i n$. (13) par rapport à la floraison d'Angelica archangelica $L$.

FIG. 1. - Density of Syrphidae (1), Muscidae and Calliphoridae (2), Vespa germanica L. (3), Andrena minutula $K$. (5), Halictus maculatus Smith (8) and Apis mellifera ligustica Spin. (13) in relation to flowering of Angelica archangelica $L$.

$\mathrm{x}$-axis : day and month.

y-axis : left : number of insects on $10 \mathrm{~m}^{2}$. right : state of flowering $(\%)$. 
tionnelle intensité le 14 mai $\left(250 / 10 \mathrm{~m}^{2}\right)$. Les mouches représentent $22,18 \%$ des pollinisateurs et une densité moyenne de 42,30 butineuses.

$V$ espa germanica $\mathrm{L}$. présente des pointes de remarquable intensité seulement les 12 et 14 mai et la fréquence de visites est vraiment médiocre ( 8 jours sur 20); elle représente $16,81 \%$ des pollinisateurs d'Angelica et sa densité moyenne est de 12,50 butineuses.

Andrena minutula $\mathrm{K}$. est l'insecte le plus représentatif ( $100 \%$ fréquence, représentativité $32,70 \%$, densité moyenne 20,40 ).

Apis mellifera ligustica Spin. montre un intérêt discontinu (fréquence de visites 17 jours sur 20 et intensité des visites acceptable dans la période du 2 au 8 mai et du 16 au 20 mai. La raison de ce comportement est que l'insecte est extrêmement polylectique, préférant butiner en même temps et avec une assiduité remarquable sur d'autres espèces (Tab. 1). L'abeille représente $17,4 \%$ des insectes pollinisateurs d'Angelica (densité moyenne 12,55).

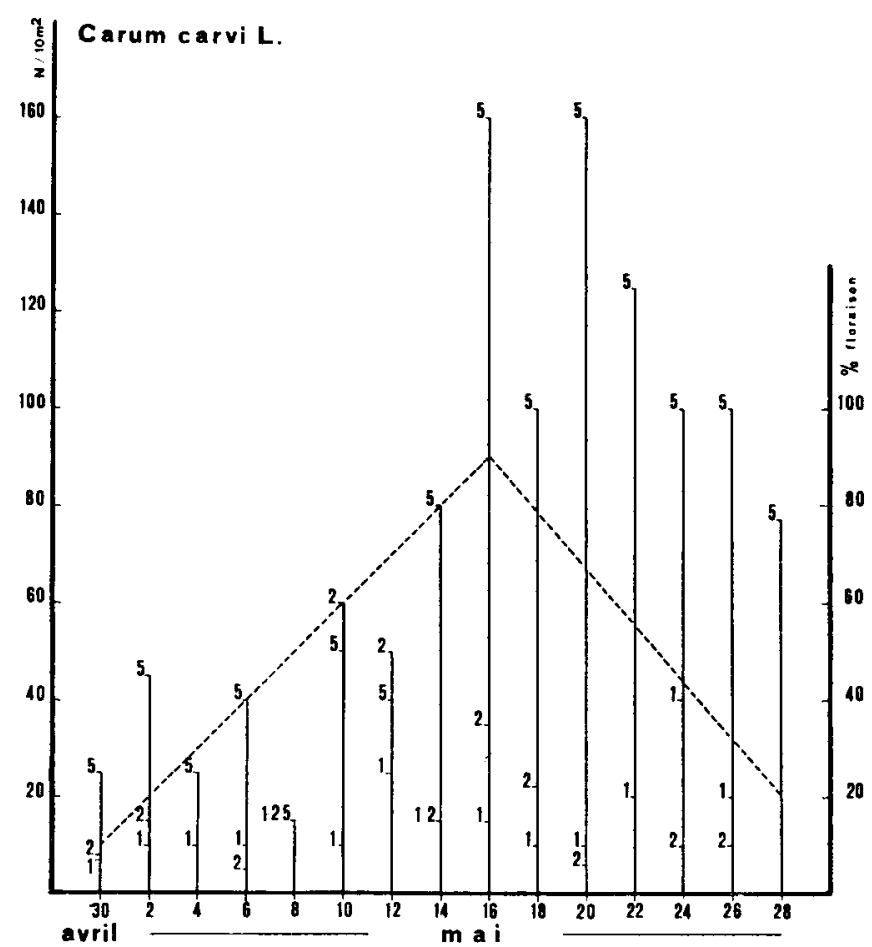

FIG. 2. - Densité de Syrphidae (1), Muscidae et Calliphoridae (2) et Andrena minutula K. (5) par rapport à la floraison de Carum carvi $L$.

FIG. 2. - Density of Syrphidae (1), Muscidae and Calliphoridae (2) and Andrena minutula K. (5) in relation to flowering of Carum carvi $L$.

$\mathrm{x}$-axis : day and month.

$y$-axis : left : number of insects on $10 \mathrm{~m}^{2}$.

right : state of flowering (\%). 
On a remarqué aussi des visites sporadiques d'Andrena flavipes Panz., Halictus maculatus Smith et Halictus scabiosae Rossi (représentativité médiocre : $4,61 \%$ ). On peut considérer que les insectes sauvages sont les meilleurs pour assurer la fécondation croisée d'Angelica archangelica (représentativité 77,70\%).

2. Carum carvi L. (surface totale $12 \mathrm{~m}^{2}$ ) (Fig. 2-3)

Les Syrphidae (Fig. 2) jouent un rôle satisfaisant, probablement attirés par les arômes de Carum (fréquence 13 jours sur 15, représentativité 11,65\%, densité moyenne 14,30). Le comportement des Muscidae et Calliphoridae est semblable (fréquence 11 jours sur 15 , représentativité $15,70 \%$, densité moyenne 16,60 ).

Andrena minutula est sûrement le pollinisateur le plus efficace sur Carum avec une fréquence de visite exceptionnelle, une représentativité très remarquable $(61,50 \%)$ et une densité moyenne de 76,20 butineuses. Halictus maculatus (Fig. 3) joue un rôle fort intéressant même s'il est très discontinu (fréquence 9 jours sur 15). Efficace surtout dans la phase finale de la floraison, il représente $10,1 \%$ des pollinisateurs et a une densité moyenne de 13,80 butineuses.

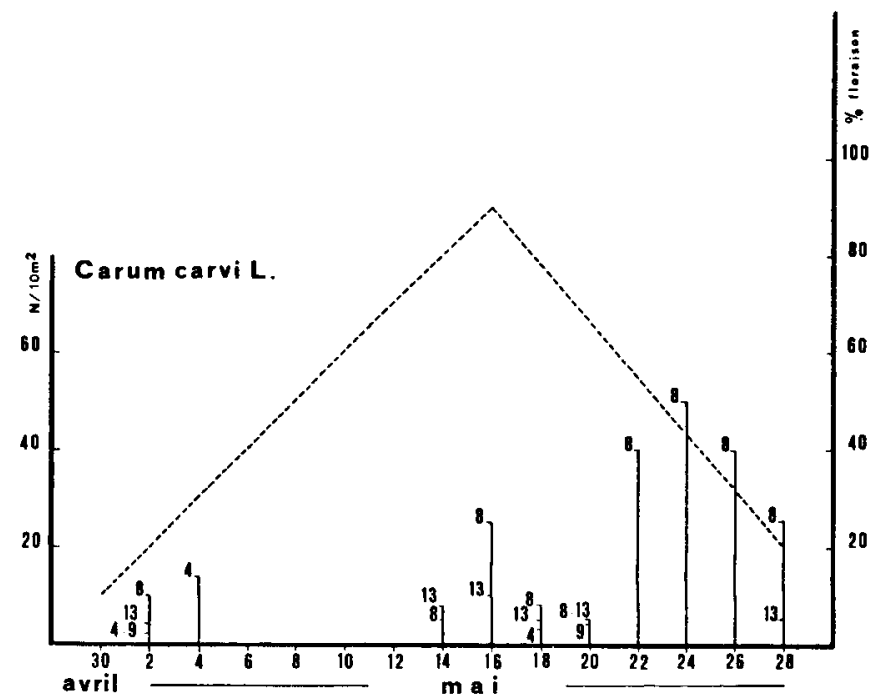

Fig. 3. - Densité d'Andrena flavipes Panz. (4), Halictus maculatus Smith (8),

Halictus scabiosae Rossi (9) et Apis mellifera Iigustica Spin. (13) par rapport à la floraison de Carum carvi $\mathrm{L}$.

Fig. 3. - Density of Andrena flavipes Panz. (4), Halictus maculatus Smith (8),

Halictus scabiosae Rossi (9) and Apis mellifera ligustica Spin. (13) in relation to flowering of Carum carvi $L$.

$\mathrm{x}$-axis : day and month.

$y$-axis : left : number of insects on $10 \mathrm{~m}^{2}$.

right : state of flowering (\%). 


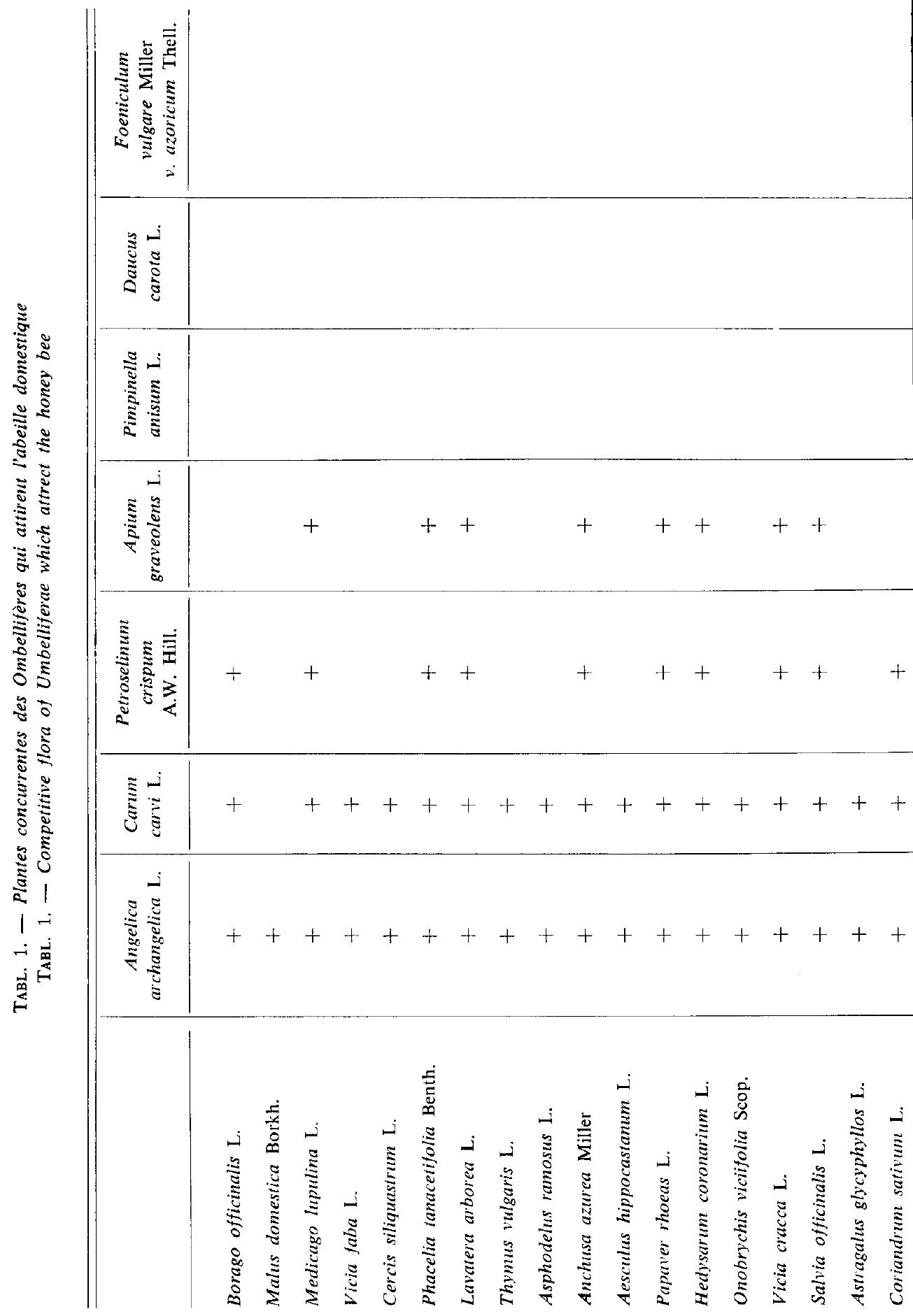




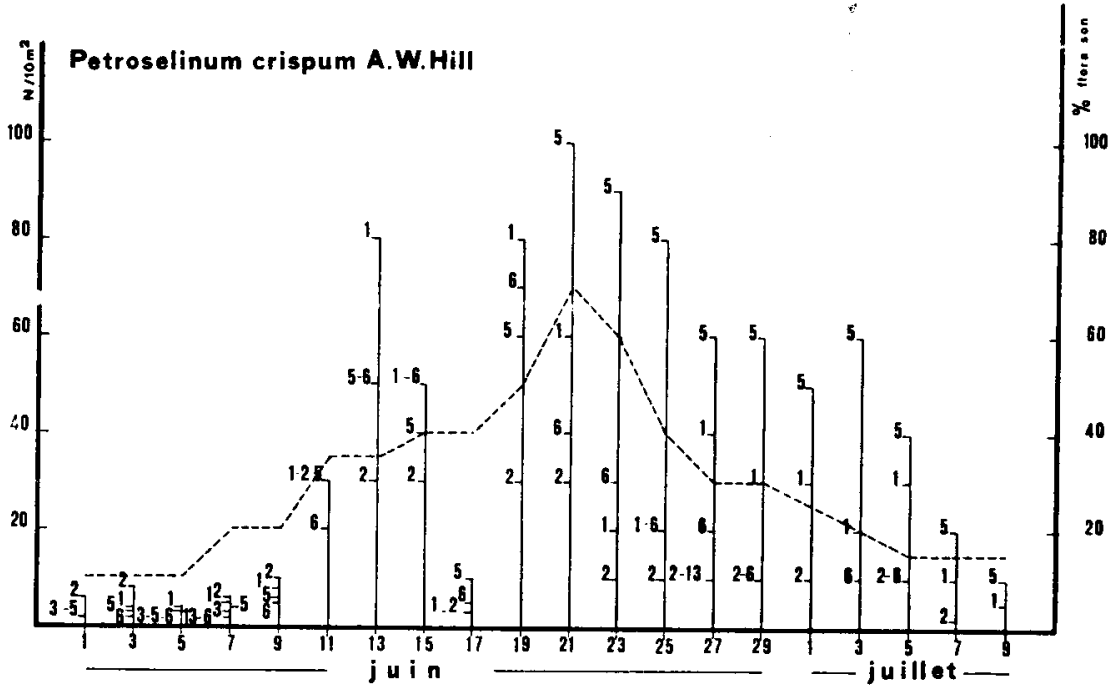

Fig. 4. - Densité de Syrphidae (1), Muscidae et Calliphoridae (2), Vespa germanica L. (3), Andrena minutula $K$. (5), Andrena ovatula $K$. (6) et Apis mellifica ligustica Spin (13) par rapport à la floraison de Petroselinum crispum A.W. Hill.

Fig. 4. - Density of Syrphidae (1), Muscidae and Calliphoridae (2), Vespa germanica L. (3), Andrena minutula $K$. (5), Andrena ovatula $K$. (6) and Apis mellifera ligustica Spin (13) in relation to flowering of Petroselinum crispum A.W. Hill.

$\mathrm{x}$-axis : day and month.

$y$-axis : left : number of insects on $10 \mathrm{~m}^{2}$.

right : state of flowering $(\%)$.

Apis mellifera ligustica joue un rôle non satisfaisant par la présence des espèces botaniques très attractives.

3. Petroselinum crispum A.W.Hill. (Surface totale $15 \mathrm{~m}^{2}$ ) (Fig. 4)

Les Syrphidae peuvent être considérés comme de bons pollinisateurs parce qu'ils montrent sur le persil une fréquence de visites remarquable (19 jours sur 20 ), une représentativité considérable $(27,73 \%)$ et une bonne densité moyenne $(26,45)$.

Les Muscidae et Calliphoridae sont moins importants bien qu'assez assidus (fréquence 17 jours sur 20 et densité moyenne 16,75).

Andrena minutula est l'insecte le plus représenté $(40,72 \%)$ avec une fréquence de visites très élevée $(100 \%)$ et une densité moyenne de 38,85 butineuses.

Andrena ovatula $\mathrm{K}$. est une espèce intéressante bien que peu assidue (fréquence 16 jours sur 20 , représentativité $18,13 \%$, densité 17,35 ). 
Apis mellifera ligustica n'est pas attirée par l'espèce et préfère s'orienter vers d'autres essences (Tab. 1).

Les pollinisateurs sauvages sont donc les meilleurs pollinisateurs du persil (représentativité $99,84 \%$ ).

4. Apium graveolens $\mathrm{L}$. (Surface totale $15 \mathrm{~m}^{2}$ ) (Fig. 5)

Les Syrphidae se montrent des habitués très assidus parce qu'ils sont attirés par les arômes du céleri. Ils manifestent une représentativité de 20,98\% et une densité moyenne de 24,05 .

Les Muscidae et Calliphoridae ont un comportement presque semblable.

Andrena minutula est le pollinisateur le plus important (fréquence de visites 15 jours sur 17 , densité moyenne 73,80 ).

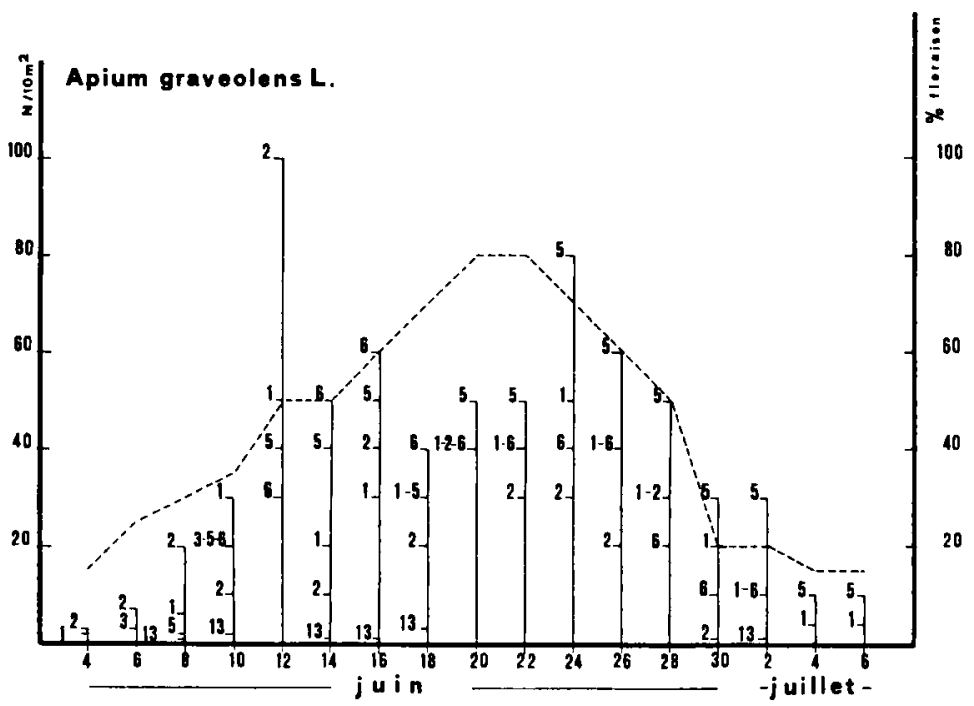

Fig. 5. - Densité de Syrphidae (1), Muscidae et Calliphoridae (2), Vespa germanica (3), Andrena minutula $K$. (5), Andrena ovatula $K$. (6) et Apis mellifera ligustica Spin. (13) par rapport à la floraison de Apium graveolens $L$.

Fio. 5, -- Density of Syrphidae (1), Muscidae and Calliphoridae (2), Vespa germanica L. (3), Andrena minutula $K$. (5), Andrena ovatula $K$. (6) and Apis mellifera ligustica Spin. (13) in relation to flowering of Apium graveolens $L$.

x-axis : day and month.

$y$-axis : left : number of insects on $10 \mathrm{~m}^{2}$.

right : state of flowering (\%). 
Andrena ovatula représente $22,66 \%$ des pollinisateurs avec une fréquence de visites peu élevée (12/17) et une densité moyenne de 19,06 butineuses ; par contre l'abeille domestique n'est pas attirée par le céleri (Tab. 1).

Les pollinisateurs sauvages avec une représentativité globale de 99,47\% sont les meilleurs pour la production de graines de céleri.

\section{Pimpinella anisum L. (Surface totale $15 \mathrm{~m}^{2}$ ) (Fig. 6)}

Les Syrphidae jouent un rôle remarquable, leur représentativité est satisfaisante $(19,53 \%)$ et leur densité moyenne $(23,60)$. Par contre les Muscidae et Calliphoridae se sont montrés peu actifs.

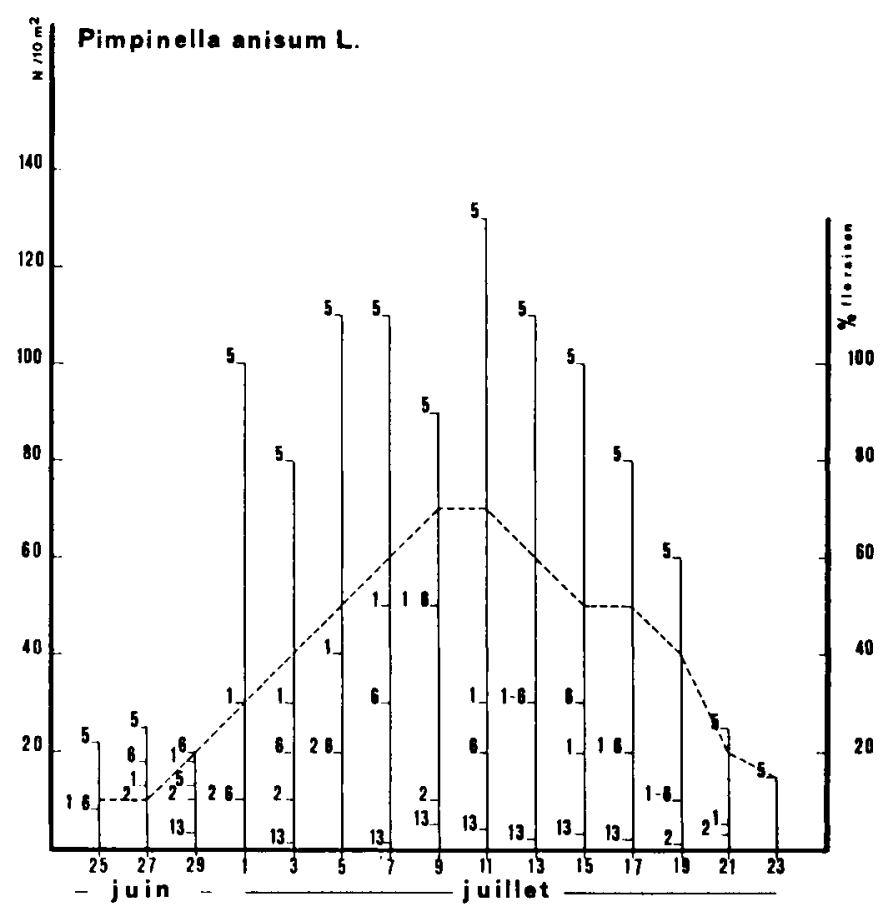

FIG. 6. - Densité de Syrphidae (1), Muscidae et Calliphoridae (2), Vespa germanica L. (3), Andrena minutula $K$. (5), Andrena ovatula $K$. (6) et Apis mellifera ligustica Spin. (13) par rapport à la floraison de Pimpinella anisum $L$.

FIg. 6. - Density of Syrphidae (1), Muscidae and Calliphoridae (2), Vespa germanica L. (3), Andrena minutula $K$. (5), Andrena ovatula $K$. (6) and Apis mellifera ligustica Spin. (13) in relation to flowering of Pimpinella anisum $L$.

$\mathrm{x}$-axis : day and month.

$y$-axis : left : number of insects on $10 \mathrm{~m}^{2}$.

right : state of flowering $(\%)$. 
Andrena minutula visite l'espèce avec une fréquence très élevée (100\%) et des intensités considérables $(5,11$ et 13 juillet). A. minutula montre une représentativité élevée $(58,74 \%)$ et une densité moyenne de 73,80 .

Andrena ovatula est moins importante qu' $A$. minutula mais joue toutefois un rôle satisfaisant. Apis mellifera ligustica n'est pas attirée par l'anis (Tab. 1).

Les insectes sauvages, avec une représentativité totale de $98,12 \%$ sont les meilleurs pollinisateurs de l'anis.

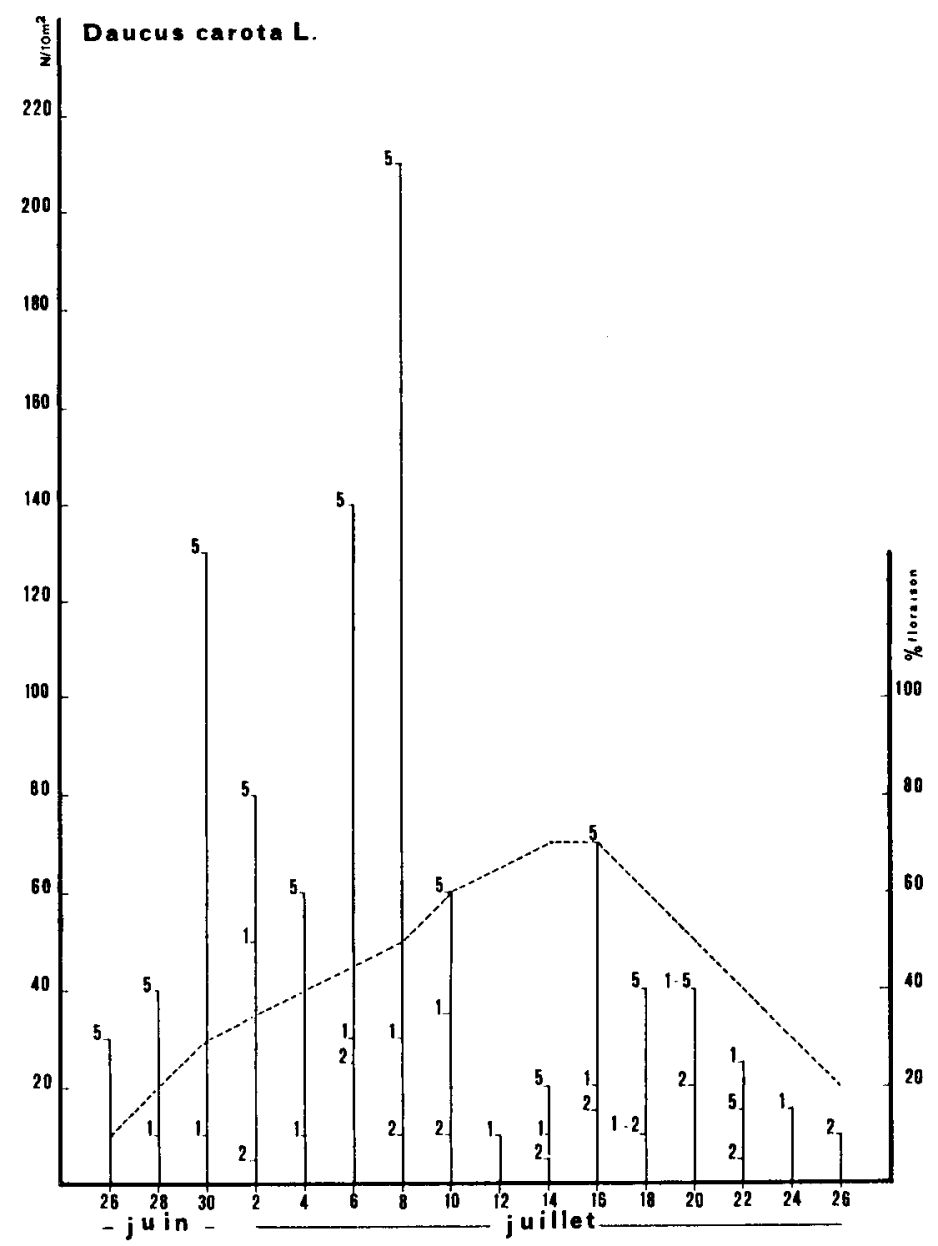

FIG. 7. - Densité de Syrphidae (1), Muscidae et Calliphoridae (2) et Andrena minutula $K$. (5) par rapport à la floraison de Daucus carota $L$.

Fig. 7. - Density of Syrphidae (1), Muscidae and Calliphoridae (2) and Andrena minutula K. (5) in relation to flowering of Daucus carota $L$.

$\mathrm{x}$-axis : day and month.

y-axis : left : number of insects on $10 \mathrm{~m}^{2}$. right : state of flowering (\%). 
6. Daucus carota L. (Surface totale $12 \mathrm{~m}^{2}$ ) (Fig. 7-8)

Andrena minutula (Fig. 7) est l'insecte pollinisateur le plus assidu avec une fréquence de visites élevée (13 jours sur 16), une représentativité de 61,55\% et une densité moyenne de 19,06.

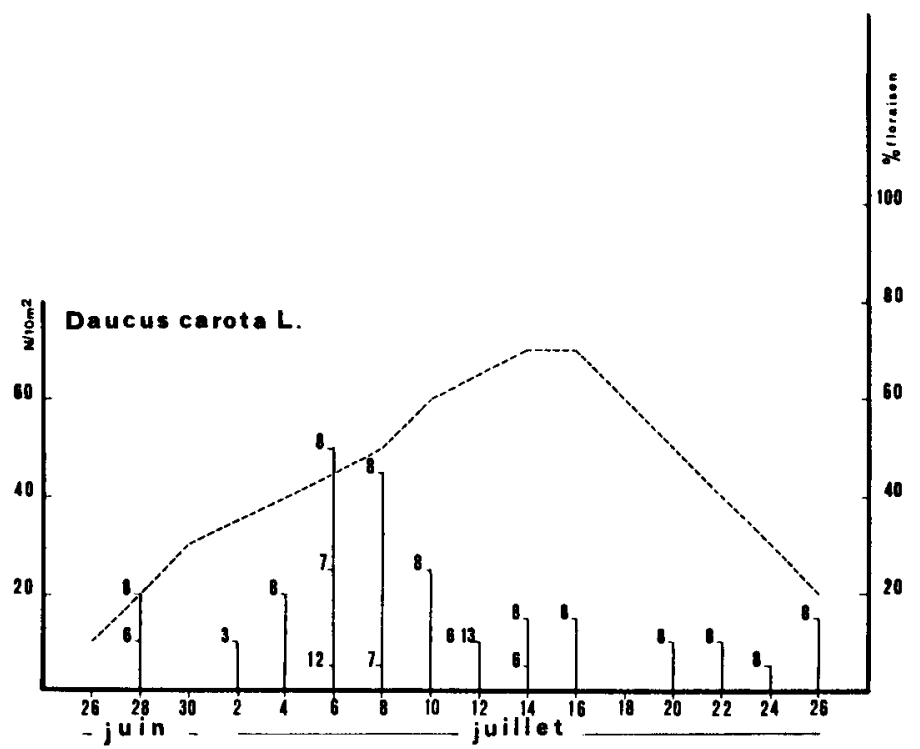

FIG. 8. - Densité de Vespa germanica L. (3), Andrena ovatula K. (6), Halictus fulvipes Klug (7), Halictus maculatus Smith (8), Anthidium manicatum L. (12)

et Apis mellifica ligustica Spin (13) par rapport à la floraison de Daucus carota $\mathrm{L}$.

FIG. 8. - Density of Vespa germanica L. (3), Andrena ovatula K. (6), Halictus fulvipes Klug (7), Halictus maculatus Smith (8), Anthidium manicatum L. (12) and Apis mellifera ligustica Spin. (13) in relation to flowering of Daucus carota $L$.

$\mathrm{x}$-axis : day and month.

$y$-axis : left : number of insects on $10 \mathrm{~m}^{2}$. right : state of flowering (\%).

Halictus maculatus (Fig. 8) avec une fréquence considérable (14 jours sur 16) contribue à la fécondation de la carotte. Sa représentativité atteint 17,03\% ; par contre Apis mellifera ligustica ignore la carotte.

On a remarqué de rares visites de Syrphidae, Muscidae, Calliphoridae, Vespa germanica, Andrena ovatula, Halictus fulvipes Klug, Anthidium manicatum L. avec une représentativité de $21,21 \%$ et une densité moyenne de 7,18 butineuses.

Les pollinisateurs sauvages sont de toute façon les meilleurs pour la carotte avec une représentativité totale de $99,80 \%$. 
7. Foeniculum vulgare Miller v. azoricum Thell (Surface totale $16 \mathrm{~m}^{2}$ ) (Fig. 9-10)

Andrena minutula (Fig. 10) est l'insecte pollinisateur le plus efficace (fréquence $100 \%$, représentativité $33,00 \%$, densité moyenne 17,6 ).

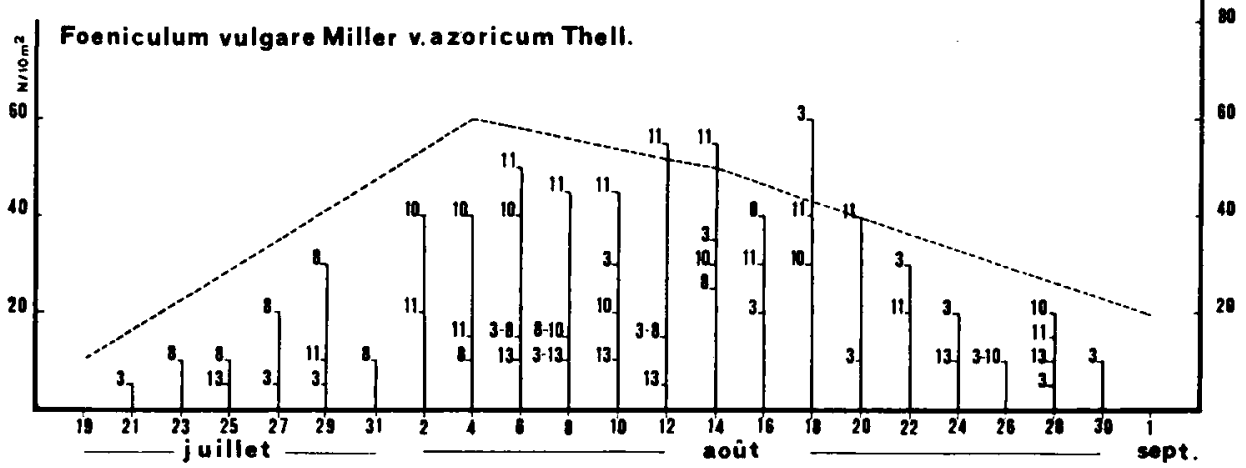

Fig. 9. - Densité de Vespa germanica L. (3), Andrena flavipes Panz. (4), Halictus maculatus Smith (8), Lasioglossum interruptum Panz. (11) et Apis mellifera ligustica Spin. (13) par rapport à la floraison de Foeniculum vulgare Miller $v$. azoricum Thell.

FIG. 9. - Density of Vespa germanica L. (3), Andrena flavipes Panz. (4),

Halictus maculatus Smith (8), Lasioglossum interruptum Panz. (11) and Apis mellifera ligustica Spin. (13)

in relation to flowering of Foeniculum vulgare Miller v. azoricum Thell.

$\mathrm{x}$-axis : day and month.

$y$-axis : left : number of insects on $10 \mathrm{~m}^{2}$.

right : state of flowering $(\%)$.

Halictus vestitus Lep. (Fig. 9) montre une représentativité de 7,69\% et une densité moyenne de 19,10 ; Il est intéressant parce qu'il est oligolectique (il ne visite que Satureja hortensis L. en plus de Foeniculum).

Lasioglossum interruptum Panz. avec une fréquence de 13 jours sur 23, une représentativité de $15,40 \%$ et une densité moyenne de 10,60 peut être considéré comme un pollinisateur satisfaisant ; par contre Apis mellifera ligustica (Fig. 9) joue un rôle presque insignifiant.

Parmi les autres insectes présents sur le fenouil on note les Syrphidae (Fig. 10), les Muscidae et Calliphoridae (Fig. 10), Vespa germanica et Halictus maculatus (Fig. 9).

Le fenouil est pratiquement pollinisé par les pollinisateurs sauvages (représentativité totale de $97,95 \%$ ). 


\section{DISCUSSION ET CONCLUSION}

D'après les résultats obtenus on peut tirer les conclusions suivantes :

- Par la présence d'un grand nombre d'essences spontanées et cultivées, le milieu considéré peut donner des résultats très fiables concernant l'attractivité de plantes et leur compétitivité, surtout en ce qui concerne l'abeille domestique (Tab. 1);

- Les Diptères sont attirés non seulement par le nectar des Ombellifères, mais aussi par les arômes émis par ces plantes;

- les données montreraient que l'abeille n'aime pas les Ombellifères considérées ;

- par contre les pollinisateurs sauvages sont les meilleurs pour la production de semence des Ombellifères considérées. Les Syrphidae, les Muscidae, les Calliphoridae, Vespa germanica, Andrena flavipes, A. minutula, A. ovatula,

TABL. 2. - Représentativité et densité des pollinisateurs sur les Ombellifères

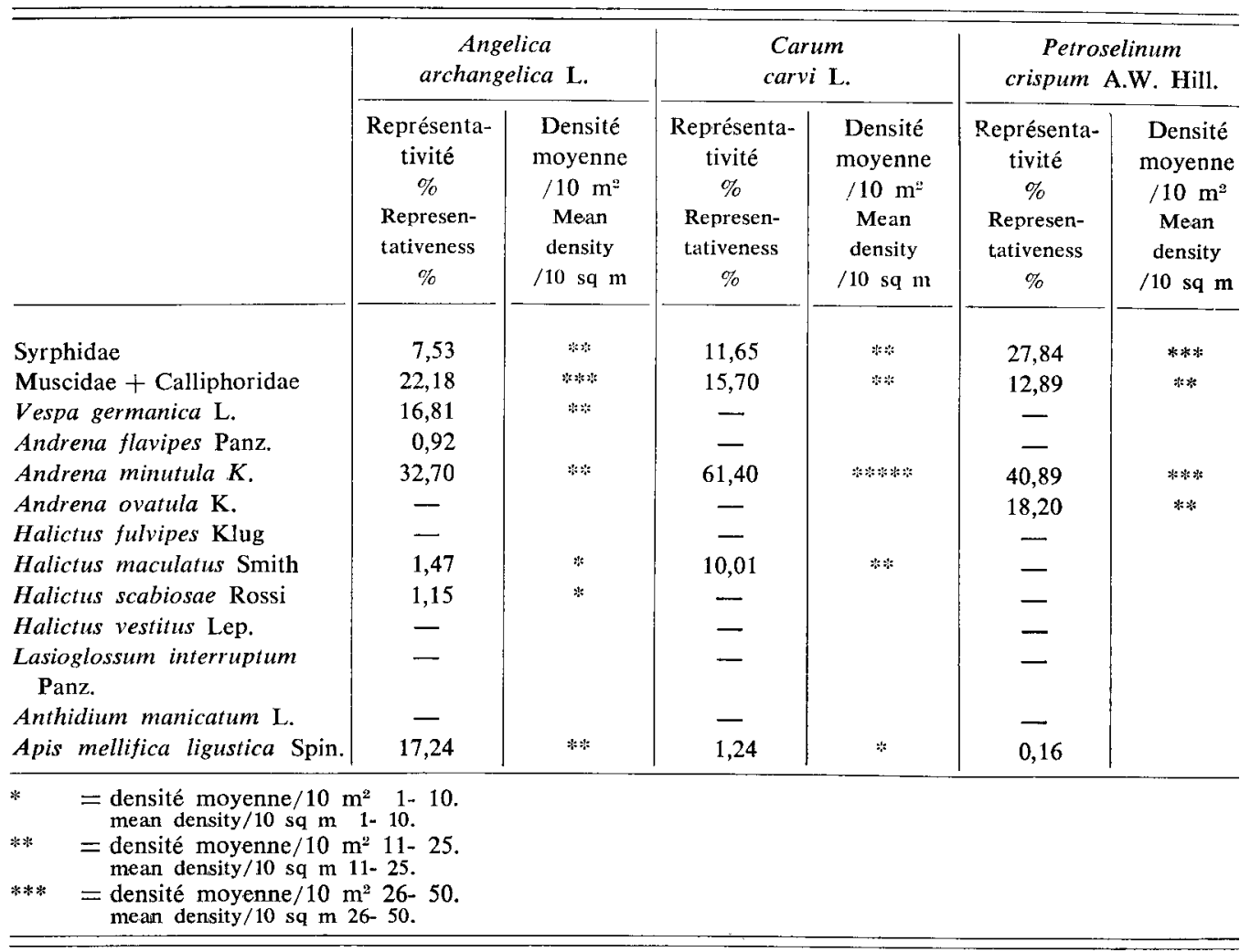


Halictus fulvipes, H. maculatus, H. scabiosae, H. vestitus, Lasioglossum interruptum, Anthidium manicatum représentent $82,76 \%$ des pollinisateurs sur Angelica, 98,76\% sur Carum, 99,84\% sur Petroselinum, 99,47 \% sur Apium, 98,12\% sur Pimpinella, 99,80\% sur Daucus et $97,92 \%$ sur Foeniculum (Tab. 2) ;

- il n'existe pas en général de corrélation positive entre le déroulement de la floraison et la densité des pollinisateurs pour chaque unité de surface; à l'exception toutefois de $A$. minutula sur Pimpinella, Petroselinum et Foeniculum et de Lasioglossum interruptum sur Foeniculum; l'absence de suivi des visites de la plupart des pollinisateurs peut s'expliquer par le fait que quelques-uns d'entre eux se sont momentanément déplacés sur d'autres espèces compétitives ;

- ces résultats, décevants pour l'abeille domestique mais très positif pour les pollinisateurs sauvages, devraient être vérifiés sur des surfaces plus grandes, où les Ombellifères en question pourraient se montrer plus attractives pour l'abeille et les pollinisateurs sauvages présenter une plus grande dispersion;

TABL. 2, - Representativeness and density of pollinating insects on Umbelliferae

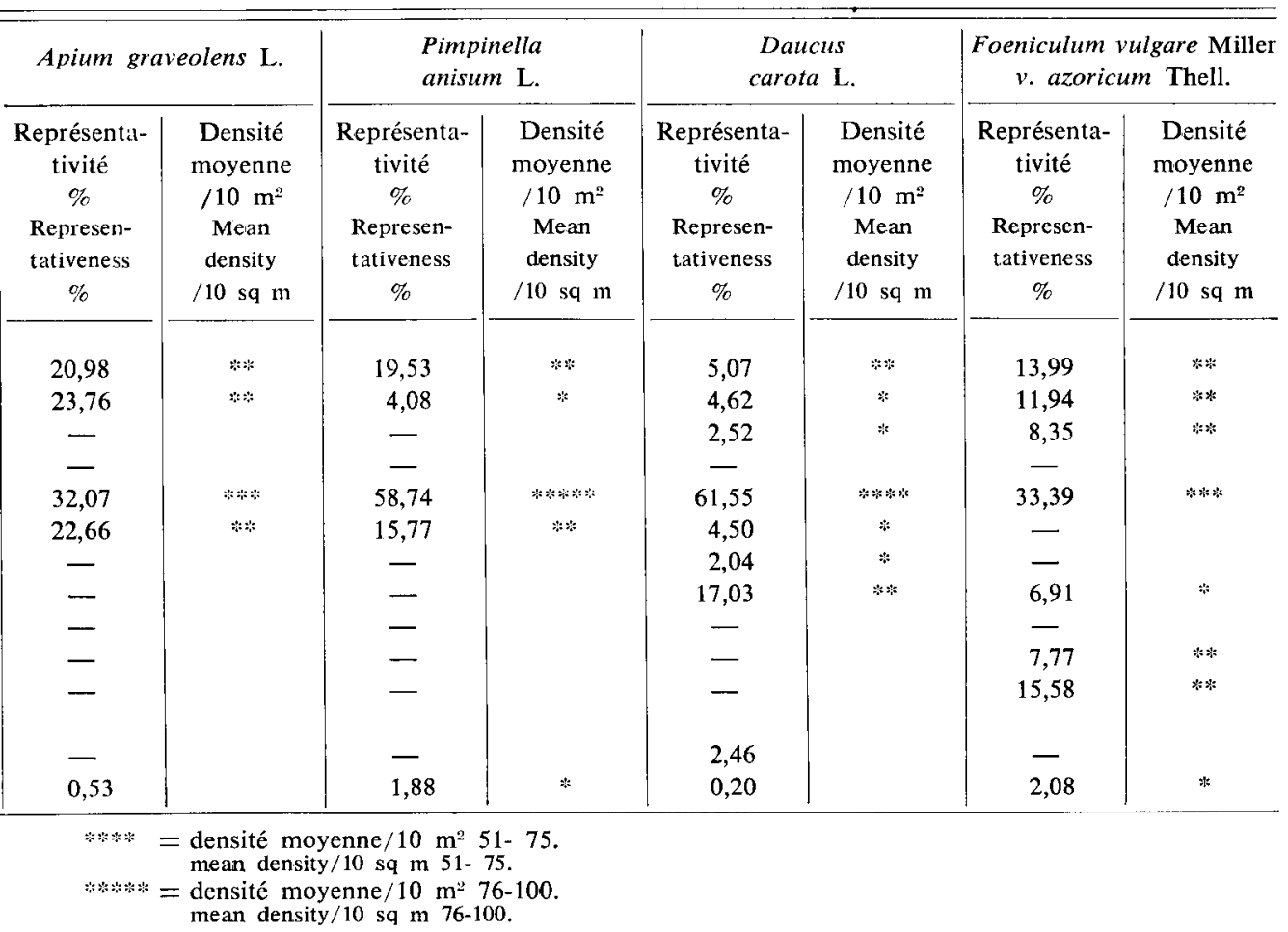




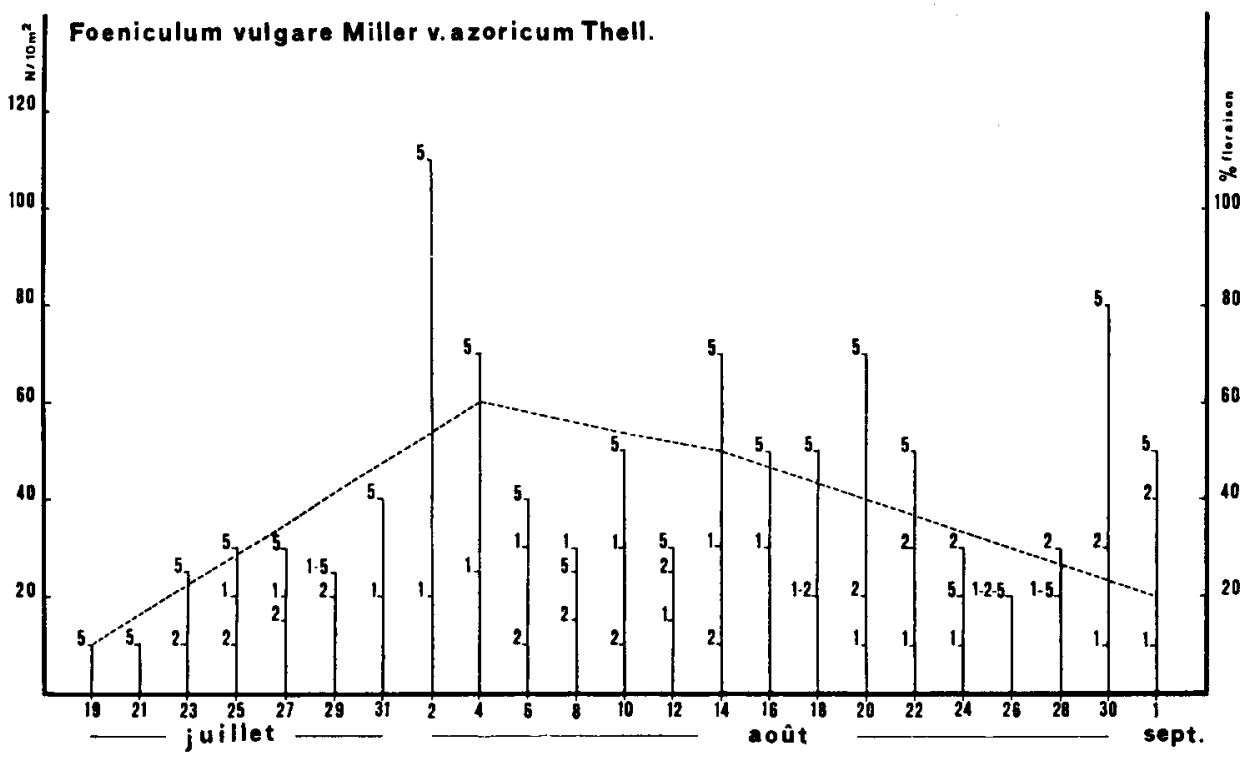

FIG. 10. - Densité de Syrphidae (1), Muscidae et Calliphoridae (2) et Andrena minutula K. (5) par rapport à la floraison de Foeniculum vulgare Miller $v$. azoricum Thell.

Fig. 10. - Density of Syrphidae (1), Muscidae and Calliphoridae (2) and Andrena minutula K. (5) in relation to flowering of Foeniculum vulgare Miller v. azoricum Thell.

$\mathrm{x}$-axis : day and month.

$y$-axis : left : number of insects on $10 \mathrm{~m}^{2}$. right : state of flowering (\%).

- le milieu étudié a néanmoins montré que de nombreuses espèces spontanées et cultivées attirent les abeilles au détriment des Ombellifères et diminuent ainsi le service que cet insecte pourrait rendre, tandis que les pollinisateurs sauvages, tout en ne dédaignant pas d'autres espèces, se montrent particulièrement fidèles aux mêmes Ombellifères.

Reçu pour publication en avril 1985.

Accepté pour publication en décembre 1985.

\section{REMERCIEMENTS}

On remercie vivement le $\mathrm{Pr}^{r}$ Pouvreau (France) et le $\mathrm{D}^{\mathbf{r}}$ Warncke (Allemagne) pour la détermination des espèces $d$ insectes, aimablement exécutée. 


\title{
SUMMARY
}

\author{
THE POLLINATING INSECTS OF SOME UMBELLIFERAE OF AGRICULTURAL \\ AND HERBAL INTEREST (ANGELICA ARCHANGELICA L., CARUM CARVI L., \\ PETROSELINUM CRISPUM A.W. HILL, APIUM GRAVEOLENS L., \\ PIMPINELLA ANISUM L., DAUCUS CAROTA L., \\ FOENICULUM VULGARE MILLER V. AZORICUM THELL.)
}

During 2 years (1982-1983) preliminary observations were carried out on the pollinating insects of some Umbelliferae of agricultural and herbal interest : angelica (Angelica archangelica), caraway (Carum carvi), parsley (Petroselinum crispum), celery (Apium graveolens), anise (Pimpinella anisum), carrot (Daucus carota) and fennel (Foeniculum vulgare $\mathrm{v}$, azoricum). These plants have been grown for several years in plots of the same dimensions (about $10 \mathrm{sq} \mathrm{m}$ ), where no pesticide treatment has been made for a long time.

In 1984, this study was continued : every two days the insects were caught and counted in order to determine the frequency and intensity of visits and also the representation of species. The observations were repeated during the whole period of flowering on small areas $\left(1 \mathrm{~m}^{2}\right)$, in different locations each time and reporting them as an area of $10 \mathrm{~m}^{2}$. Simultaneously the visits of insects on the surrounding flora were observed in order to value their attractiveness and competitiveness. The pre-eminent aim was to compare the pollinating role of wild insects with that of the honeybee.

The results indicated that the honeybee (Apis mellifera $\mathrm{L}$.), carries on a fair role as pollinator of Angelica archangelica (representativity 17,24\%), while it is practically absent on the other Umbelliferae. Consequently the wild insects (Syrphidae, Muscidae, Calliphoridae, Vespa germanica L., Andrena flavipes Panz., A. minutula K., A. ovatula K., Halictus fulvipes Klug, H. maculatus Smith, H. scabiosae Rossi, $H$. vestitus Lep., Lasioglossum interuptum Panz., Anthidium manicatum L.) are truly responsible for the cross-pollination of the Umbelliferae studied. They represent $82,76 \%$ of the pollinators present on angelica, $98,76 \%$ on caraway, $99,84 \%$ on parsley, $99,47 \%$ on celery, $98,12 \%$ on anise, $99,80 \%$ on carrot and $97,92 \%$ on fennel.

Umbelliferae are very attractive for wild pollinators even in presence of a rich alternative flora; nevertheless the role of such pollinators should be verified on greater areas in which the visitation intensity would probably suffer a considerable decrease because of the dispersion of pollinators. Since the honeybee is a polylectic insect, its exploitation as pollinator is hypothetically possible only when competitive floral are absent and by increasing the number of hives per ha to a great extent.

\section{ZUSAMMENFASSUNG}

DIE BESTAUBENDEN INSEKTEN VON EINIGEN UMBELLIFEREN MIT BEDEUTUNG FÜR DIE LANDWIRTSCHAFT

UND FÜR HEIL- UND GEWÜRZPFLANZEN

(ANGELIKA, KüMMEL, PETERSILIE, SELlERIE, ANIS, KAROTTE UND FENCHEL)

Während einer Periode von zwei Jahren (1982-1983) wurden Beobachtungen über die bestäubenden Insekten von den im Titel genannten Umbelliferen angestellt. Diese Pflanzen wurden während mehrerer Jahre auf gleichgroßen Beeten (etwa $10 \mathrm{~m}^{2}$ ) gezogen und nie mit Pestiziden behandelt.

Im Jahre 1984 wurde die Untersuchung fortgeführt : Alle zwei Tage wurden die Insekten gefangen und gezählt, um die Häufigkeit und Stärke der Besuche und auch die Repräsentanz zu erfassen. Die Beobachtungen wurden während der ganzen Blütezeit auf kleinen Flächen $\left(1 \mathrm{~m}^{2}\right)$ fortgesetzt, wobei die Flächen ständig gewechselt und schließlich auf $10 \mathrm{~m}^{2}$ umgerechnet wurden. 
Gleichzeitig wurde der Insektenbesuch auf der umgebenden Flora registriert, um ihre Attraktivität und ihren Konkurrenzwert zu bestimmen. Das Hauptziel war der Vergleich der Rolle von Wildinsekten mit der von Honigbienen.

Die Ergebnisse zeigten, daß die Honigbienen (Apis mellifera L.) bei der Bestäubung von Angelica archangelica eine gewisse Rolle spielten (Anteil 17,24\%), während sie bei den anderen Umbelliferen praktisch fehlte. Deshalb sind Wildinsekten (Syrphiden, Musciden, Calliphoriden, Vespa germanica L., Andrena flavipes Panz., A. minutula K., A. ovatula K., Halictus fulvipes Klug, H. maculatus Smith, H. scabiosae Rossi, H. vestitus Lep., Lasioglossum interruptum Panz., Anthidium manicatum L.) tatsächlich für die Kreuzbestäubung der untersuchten Umbelliferen verantwortlich. Sie bildeten $82,76 \%$ der Bestäuber auf Angelika, 98,76\% auf Kümmel, 99,84\% auf Petersilie, $99,47 \%$ auf Sellerie, $98,12 \%$ auf Anis, 99,80\% auf Karotte und $97,92 \%$ auf Fenchel.

Umbelliferen sind selbst dann für Bestäuber aus der Wildfauna sehr attraktiv, wenn eine reiche Alternativ-Flora vorhanden ist; trotzdem sollte die Rolle dieser Bestäuber in einem größeren Areal bestätigt werden, wo die Besuchsintensität wahrscheinlich durch die verstreutere Verteilung der Bestäuber eine beträchtliche Veränderung erfahren würde. Die Honigbiene, ein Musterbeispiel eines polylektischen ( $=$ vielseitigen) Insekts, zieht es vor, an konkurrenzfähigeren Pflanzen zu sammeln. Deshalb wäre ihr Einsatz als Bestäuber hypothetisch nur dann möglich, wenn die Konkurrenzflora fehlt und die Zahl der Bienenvölker per ha wesentlich vergrößert wird.

\section{REFERENCES BIBLIOGRAPHIQUES}

BoharT G.E., NyE W.P., 1960. - Insect pollinators of carrots in Utah. Bull. Ulah. Agric. Exp. Stn., $\mathrm{n}^{\circ} 419,16$ p.

Borthwick H.A., Enswelter S.L., 1933. - Carrot breeding experiments. Proc. Am. Soc. hortic. Sci., 30, 531-533.

Erickson E.H., Peterson C.E., 1978. - Problems encountered in the pollination of cytoplasmically male-sterile hybrid carrot seed parents. Proc. IVth Int. Symp. on Pollination, Md. Agric. Exp. Stn. Spec. Misc. Publ. 1, 59-63.

FREE J.B., 1970. - Insect pollination of crops. Academic Press London, 314-317.

Hawtorn L.R., Bohart G.E., Toole E.H., 1956. - Carrot seed yield and germination as affected by different levels of insect pollination. Proc, Am. Soc. hortic. Sci., 67, 384-389.

Hawtorn L.R., Bohart G.E., Toole E.H., Nye W.P., Levin M.D., 1960 - Carrot seed production as affected by insect pollination. Bull. Utah Agric. Exp. Stn., n ${ }^{\circ} 422,18$ p.

Narajanan E.S., Sharma P.L., Phadke K.G., 1960. — Studies on requirements of various crops for insect pollinators I. Insect pollinators of saunf (Foeniculum vulgare) with particular reference to the honeybees at Pusa (Bihar) Indian Bee J., 22, 7-11.

Pankratova E.P., 1958. - Data on the biology of blossoming and pollination of carrots. Dokl. Tskha, 36, 118-123 (en Russe).

Pesson P., Louveaux J., éd. 1984. - Pollinisation et productions végétales. I.N.R.A., Paris, 476-480.

Ricciardelli D'Albore G.C., 1983. - Ethologie florale de quelques insectes pollinisateurs. Ve Symposium International sur la pollinisation. Versailles 1983. I.N.R.A., 381-386.

Ricciardelli D’Albore G., D'Ambrosıo M., 1979. - Prime osservazioni sull'attività impollinatrice dell'ape (Apis mellifera ligustica Spin.) e di altri pronubi sul Coriandolo (Coriandrum sativum L.). Apic. Mod., 5, 151-157.

Schmiedeknecht O., 1930. - Die Hymenopieren Nord und Mittel-Europas. - Jena, Gustav Fischer, $1062 \mathrm{p}$.

Zangheri P., 1976. - Flora italica. - CEDAM, Padova. 